\title{
Europhysics Conference Reports
}

\section{Optical Systems and Applications}

The 1980 European Conference on Optical Systems and Applications which was held in Utrecht, 23-25 September, 1980, is a biennial conference that came into being through a merging of the European ElectroOptics and Ecosa Conferences. The aim of the organizers was to provide scientists and engineers with a platform where they can discuss their problems in the field of optical systems (including both engineering and applications) and expose their achievements. They should feel gratified by the response to their efforts.

The Conference attracted about 350 participants (many of whom expressed a positive reaction afterwards); 120 papers were presented, and in the evening, poster sessions were held which had, in part, a social character that stimulated lively discussions. Apart from the standard subjects such as non-linear optics, anemometry, optical metrology, the scientific programme included sessions on medical and biological applications and one that focused on industry in which exhibitors could give a scientific account on some of their products.

The exhibition accompanying the Conference contained 30 stands. A number of new products were on show and both attendance and business were above expectations. Particularly interesting were the mini $\mathrm{CO}_{2}$ laser of Ferranti and the ring laser stabilizer of Spectra Physics.

In an invited paper, Huignard (ThomsonC S F) described work on adaptive optics by phase conjugation in $\mathrm{Bi}_{12} \mathrm{SiO}_{20}$ ( $\mathrm{B} \mathrm{S} \mathrm{O}$ ) non-linear electro optic crystals. High resolution and sensitivity with relatively long storage times $(\sim 30 \mathrm{~h})$ were obtained. The electric field bias adds an extra dimension in time-dependent applications. Tiziani (Stuttgart) used these crystals for real time holographic vibration and deformation analyses as an extension to previous speckle techniques.

Andersen (Texas Techn. Univ.) described an efficient way of infared detection by conversion up to the visible in doped $\mathrm{GaP}$, and Koch (Hannover) talked on colour centre lasers in a wavelength region that was not available until now $(0.8-1.8 \mu \mathrm{m})$. It would be interesting to have a comparison between the merits of these lasers and those using transition metal ions, such as $\mathrm{Co}^{2+}, \mathrm{Ni}^{2+}$, etc.

An ingenious method for measuring micro vibrations down to $1 \mathrm{~nm}$, in-plane and out-of-plane, by heterodyne speckle interferometry was presented by Dändliker (Neuchatel) (Fig. 1) while Collins and Hulme (R.S.R.E.) discussed well-engineered range and velocity meters using a mini on ocean waters. babilities are achieved.

$\mathrm{CO}_{2}$ laser (c.w.). Included was a heterodyne system where the laser is modulated with a chirp wave-form centred on $60 \mathrm{MHz}$, generated by a surface acoustic wave dispersive filter. Airborne fluorescence Lidar systems (Gehlhaar, Oldenburg) were shown to be suitable for detection of fluorescent layers (oil, tracers, chlorophyll)

There was much interest in the sessions on medical and biological applications. The papers were all of high standard, but one might mention laser coagulation of dermal lesions described by van Gemert (Eindhoven) and microfluorimetry with time resolution by Sacchi (Milan).

Anemometry and particle sizing have become full grown laser application fields for which subtle techniques and measurements were described by Pusey, Pike (R.S.R.E.) and Schulz-Dubois (Kiel). The present state of the art in semiconductors lasers was well described by Maslowski (A.E.G., Ulm) and that in optical recording by Carasso (Philips, Eindhoven). By skilful coding high bit densities and low error pro-

The application of optical fibres as sensors of current, voltage, temperature and mechanical stresses and vibrations has

\section{Nuclear Methods for Solid State Physics and Materials Research.}

\section{Based on contributions from H. Jex and G. Mauck (Frankfurt)}

In these days of highly specialized conferences covering restricted fields, it is unusual to find one that is concerned with such apparently diverging interests as materials research, solid state physics and nuclear physics. This was, nevertheless, the background to the "Europhysics Conference on Nuclear Physics: Methods in Materials Research" that was held in Darmstadt, 23-26 September under the auspices of the Nuclear Physics Division, with K. Bethge of the Johann Wolfgang GoetheUniversität, Frankfurt am Main, chairing the organizing committee.

The Conference, which it is hoped will have the long term effect of stimulating collaboration between the different groups of physicists represented, attracted the participation of approx. 180 materials scientists, solid state and nuclear physicists with a good attendance from eastern European countries and a sprinkling from farther field. In the four morning sessions, 19

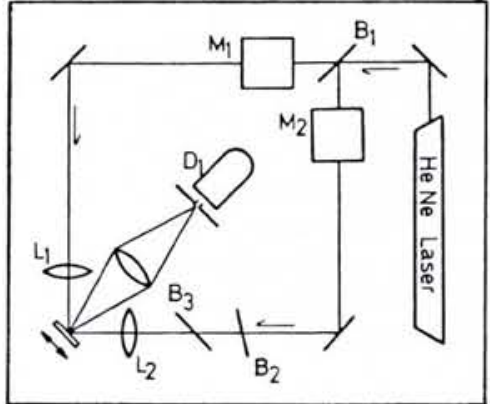

Fig. 1 - System arrangement for measuring inplane displacements.

become an important field of study. In lowloss fibres, the interaction length can be made very large but ingenuity is required to measure only the parameter desired. Ulrich (Hamburg) gave an overview and Smith and Rodgers (Leatherhead) covered some field-tested examples.

The above is merely a sample of a most impressive collection of papers. No mention is made for example of the non-linear work of Smith's group (Edinburgh), laser annealing (von Allmen, Bern), V.L.P. mastering (Olijhoek, Philips) and other important results that were presented. For those interested in more information, the abstracts of the papers are available as Europhysics Conference Abstracts, Volume 41.

\section{B. Bölger, Eindhoven}

invited papers were presented, and on three afternoons, 67 contributed papers were discussed in poster sessions; the abstracts appear in ECA Volume $4 \mathrm{~K}$ and the Proceedings will be published by Vieweg-Verlag, Wiesbaden in January.

The tone of the Conference was set early on, as being concerned with practical problems and with the delineation of those areas of research where solutions are of importance to materials scientists. There seemed to be a consensus that the key areas concerned both the structure and microstructure and comprised:

- phases, their crystal structure and chemistry,

- phase boundaries,

- grain boundaries within phases,

- precipitates within phases, their crystal structure and lattice coherence within the host lattice,

- precipitates at boundaries,

- solute segregation at boundaries, 
- dislocation structure within phases,

- free surfaces, their segregation patterns and covering films,

- cracks.

It was convincingly shown, that measurements of elemental and chemical composition in microstructures, as well as of their physical state are basic goals of materials research in the future.

The view of the opening speaker, who stressed the need for crack characterization, was that independent measurement by different techniques and in situ was the key to making progress in our understanding of much materials behaviour and notably failure mechanisms. Cracks play the major role in the ultimate failure of materials, either under static or cyclic conditions. They include very tiny cracks at the initial stage, as well as large cracks extending over several grains and resulting from the growth of small cracks, or surface imperfections. It is of prime importance to detect these cracks, measure their lengths, shape and growth rate. These observations and measurements have to be performed even in fundamental work, not only under simple experimental conditions, but also under operational conditions. Despite many interesting developments (mainly confined to metals) there are still practical difficulties when studying metallic specimens in complex environments, and even more when dealing with ceramics. New detection methods are needed for both better crack characterization, and the detection of cracks in industrial structures and equipment such as engines, aircraft, shafts, nuclear reactors, not to mention orthopoedic implants, where in-situ examination is required for safety and maintenance purposes.

Even so, fundamental studies are an essential element of progress and in some instances, techniques are limited by the need to be close to major installations. A case in point is the use of thermal and ultracold neutrons. At specialized laboratories the range of sources available in terms of momentum and energy space is wide and they have been applied to the investigation of both structure and dynamics. Techniques include: small angle scattering, which has given new insights into the physisorption of gases on to solid surfaces such as methane or ammonia on graphite, spin echo methods which have been applied to polymer solutions, and ultra cold neutron optics used to investigate various surface phenomena. Hyperfine interactions, i.e. the interactions between the nuclear moments and the extra-nuclear electromagnetic field have a short range which makes them ideal for sampling the near surroundings of nuclei. For example, perturbed angular correlations have been found to be very sensitive to the electric quadruple interaction that arises when defect formation breaks the original structural symmetry of the solid. With ${ }^{111}$ In as probe, this same technique has been successfully applied to vacancy trapping phenomena in fcc metals. The Moessbauer effect can also be used to study defects in numerous materials.

Micro-metallurgy by ion implantation lends itself to both basic and operational studies. Here the essential process is to implant ions such as nitrogen, boron, carbon, etc., in metals or alloys, in thin layers about $1 \mu \mathrm{m}$ thick. These extended thin layers are not in thermodynamic equilibrium and show new and surprising states of location, diffusion, etc. Micro-metallurgical structuring can be of importance in connection with wear, fatigue and corrosion. Applications include the study of friction coefficients and wear of nitrogen-implanted and pure materials, and the use of nitrogen implantation to increase the life of press tools.

Particle implantation is not however restricted to the production of surface layers of modified metallurgical characteristics, but is also of value as a measuring tool. Of growing interest is the work being done with very fine beams of say, $200 \mu \mathrm{m}$ diameter, associated with detection equipment such as $\mathrm{Si}$ (Li) counters which analyse the particle-induced $\mathrm{X}$-ray emission. This is the PIXE technique that has been used for a number of years and which has proved to be of particular value in the assay of filter deposits arising from air-borne pollution, and also in the analysis of biological specimens.

Particle beams have, moreover, increased the scope of tracer techniques for wear measurement. By using accelerated beams of different characteristics for the activation of parts subject to wear, the decay characteristics of the material can be made a function of depth, so that by analysing the radiation from the debris, an assessment can be made of both bulk corrosion and such effects as pitting corrosion.

Relatively new to materials study is the use of beams of muons, positrons and pions. The first two are particles with spin $1 / 2$ and exhibit both electrical and magnetic interactions whereas the pion, having zero spin, does not interact magnetically. The muon is, in practice, an ideal probe for studying the reaction of metallic electrons to a single positive "impurity" charge. In addition, it makes a powerful tool in diffusion studies where the effect of impurities can be very pronounced. It can also be used to study preferred sites for hydrogen-like interstitial atoms, and spin ordering and spin dynamics in magnetic environments.

The use of positrons has given rise to the development of a low energy diffraction technique somewhat analogous to the well
Instituut voor Kernphysisch Onderzoek

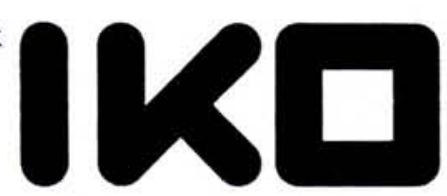

IKO, the intermediate energy nuclear physics section of the Dutch institute for nuclear and high energy physics (NIKHEF sectie $\mathrm{K}$ ), has an opening for a research associate in nuclear theory.

\section{THEORETICAL NUCLEAR PHYSICIST}

The institute has started operation of its $500 \mathrm{MeV}$ high duty cycle electron linac which will be used for research in electron scattering and low energy pion/muon physics.

The appointment is for a maximum of two years.

Applicants are expected to have experience in areas of nuclear theory which are closely related to the experimental program.

Applications including a career resume and the names of three referees should be sent to:

Justus H. Koch,

IKO, Postbus 4395,

1009 AJ AMSTERDAM, The Netherlands

(Tel. : (20) 59221 71), before 15 February, 1981. 
known LEED techniques. But the positron can also provide unique information on the electronic and physical structure of bulk materials, as well as defect and other impurity centres, from an analysis of its life time and of the angular correlation between the annihilation photons.

The availability of pion beams has further stimulated the development of devices for vacancy site and defect localization. Pions can get trapped at vacancies like muons or positrons, and their position can be determined from the decay muons, when the channelling effect of the crystal is known.

Among the most recent developments in charged particle applications has been the introduction of heavy ions into the field of materials research. Compared to other types of radiation the special characteristic of heavy ions is high damage density along well-defined tracks, the damage being confined to a channel of about $100 \AA$ diameter. The tracks are extremely straight and their length is unambiguous. This can be used to increase the storage density of magnetooptic films, to create membranes with a single submicron hole, and to produce precision filters. A particularly impressive application is that of microlithography for the fabrication of micro structures.

It is evident that nuclear physics techniques have an important role to play.

\section{Atomic Physic Division}

The new Board of the Atomic Spectroscopy Section (EGAS) of the Atomic Physics Division is as follows:

Chairman: B. Cagnac, Univ. Pierre et Marie Curie, Paris; and

Secretary: A. Dönszelmann, Zeeman Lab., Amster dam, as published in the June issue of Europhysics News.

Members:

E. Arimondo, University, Pisa

R.J. Champeau, Aimé Cotton Lab., Orsay

J. Heldt, University, Gdansk

H. Hühnermann, University, Marburg

I. Lindgren, Chalmers Univ., Göteborg

J.D. Silver, Clarendon Lab., Oxford

A.P. Thorne, Imperial College, London

R. Winkler, Tech. Univ., Berlin

\section{DIVISIONAL NEWS}

The Executive Committee has confirmed the following Divisional posts:

\author{
Astronomy and Astrophysics Division \\ Chairman: C. Cesarsky \\ SEP.CEN Saclay, BP-2 \\ F-91405 Gif-sur-Yvette \\ T. (6) 9418000 \\ Computational Group \\ Chairman: R. Zelazny \\ Institute of Nuclear Research \\ PL-Swierke k. Otwocka \\ T. 793481 - Tx. 813244 ibjsw PL
}

Nuclear Physics Division

Chairman: V. Meyer

Physik-Institut Universität

Schönberggasse 9

$\mathrm{CH}-8001$ Zurich

T. (1) 2572927

Plasma Physics Division

Vice-chairman: A. Gibso

JET Joint Undertaking

Abingdon OX14 3EA UK

T. (235) 21840

Quantum Electronics Division

Chairman: K.L. Kompa

Max-Planck-Gesellschaft

Projektgruppe für Laserforschung

D-8046 Garching bei München

T. (89) 3299-701

\section{Astronomy and Astrophysics}

The new Board of the Solar Section of the Astronomy and Astrophysics Division is as follows:

Chairman: P. Maltby, University, Oslo and

Secretary: $H$. Nussbaumer, ETH, Zurich, as published in the June issue of Europhysics News

\section{Members:}

R.M. Bonnet, Lab. of Stellar and Planetary Physics, Verrières-le-Buisson

Cl. Chiuderi, Observatory, Florence

A.D. Fokker, Astronomical Inst., Utrecht

J. Heyvaerts, Observatory, Meudon

C. Jordan, University, Oxford

M. Kuperus, Astronomical Inst., Utrecht

J.-C. Pecker, Astrophysics Inst., Paris

I.W. Roxburgh, Queen Mary Coll., London

J.O. Stenflo, Inst. of Astronomy, Zurich

C. Zwaan, Astronomical Inst., Utrecht

\section{CMD Announcement}

The Board of the Condensed Matter Division has taken the following decisions concerning the next general conferences:

- in 1982 the Conference will take place in Manchester, (UK).

Chairman: Prof. V. Heine, Cavendish Laboratory, University of Cambridge, Madingley Road,

Cambridge $\mathrm{CB} 3 \mathrm{OHE}$, UK

- in 1983 the Conference will take place in

Lausanne (Switzerland).

Chairman: Prof. E. Mooser, Institut de Physique, Université de Lausanne, Dorigny,

$\mathrm{CH}-1015$ Lausanne.

These General CMD Conferences will follow the style and form of the 1980 Antwerp General Conference. Both meetings are independent EPS ventures. All decisions concerning the committees, the programmes, which will deal with all aspects of the physics of condensed matter, etc. will be taken by the Chairmen of the Conferences on behalf of the CMD Board. It is intended to provide a European forum for current research in solid state physics and to encourage international cooperation in the field. At each Conference, there will be a series of symposia on recent developments, as well as contributed papers. Participation in the Conferences is open to all.

Future EPS-CMD Conferences will be organised by those institutions or physicists who, after due application, have been selected by the CMD Board. They will be organised as independent European initiatives and not annexed to any national meetings.

Applications to organize the General CMD Conferences for 1984 and 1985 should be sent as soon as possible to the Chairman of the Board:

Prof, J.T. Devreese, University of Antwerp (U.I.A.), Department of Physics, Universiteitsplein 1, B-2610 Wilrijk.

\section{The EPS Secretariat is closed for holidays from 24 Dec. - 4 Jan.} Happy New Year!

Europhysics News is the official journal of the European Physical Society that comprises 28 National Societies. Academies and Groups, over 3000 Individual Ordinary Members and 30 Associate Members. Governing bodies Members and 30 Associate Members. Governing bodies
of EPS are the General Meeting, Council and an elected Executive Committee responsible for detailed policy. EPS promotes the collaboration of physicist throughout Europe and encourages all aspects of international exchange in physics. EPS awards scholarships for research and studies in different countries. EPS research and studies in different countries. EPS
publishes, in addition to Europhysics News, Europhysics Conference Abstracts, Europhysics Educa tion News and the Proceedings of its General Con ferences. Individual Ordinary Members receive ferences. Individual Ordinary Members receive
Europhysics News (subscription for non-members: 75 Sw.Fr./a), substantial rebates on many publications and pay reduced fees at conferences. Application for membership is made through the permanent Secretariat in Geneva. Annual subscription for members of a Na. tional Society from 1 January, 1980 is 32 Sw. Fr.

\section{Editor: E.N. Shaw}

Meetings Compilation: W.S. Newman

Editorial Board

K. Appert, A. Baratoff, G.J. Béné,

G.R. Macleod, A. Maeder, J. Muller

Editorial and Advertising Office at the EPS Secretariat.

Address: EUROPEAN PHYSICAL SOCIETY P.O. Box 69 . CH-1213 Petit-Lancy 2 Switzerland

Telephone: Geneva (22) 931130

Telex: 23455 alarm ch

Cables: europhys genève

Printed by: Pfirter frères SA

CH-1213 Petit-Lancy/Switzerland 